\title{
Colorectal cancer risk associated with abdominal obesity. data review
}

Keywords: epidemic, colorectal cancer, cancer mortality, complex phenotype

\section{Introduction}

One of the biggest health threats around the world is obesity. Knowledge about this condition and its causes are widespread, yet the epidemic keeps rising. The association between abdominal obesity (as measured by waist circumference/WC and waist-to-hip ratio/ WHR and colorectal cancer/CRC has not been fully quantified, and the magnitude of CRC risk associated with abdominal obesity is still unclear. Beyond this relationship, there are a number of challenges posed in the context of interpreting whether being overweight (BMI $\left.25.0-29.9 \mathrm{~kg} / \mathrm{m}^{2}\right)$ or obese $\left(\mathrm{BMI}>\mathrm{or}=30.0 \mathrm{~kg} / \mathrm{m}^{2}\right)$ adversely influences disease progression, cancer mortality and survival. ${ }^{1,2}$ Body mass index/BMI is a complex phenotype that may interact with genetic variants to influence colorectal cancer risk.

\section{Waist circumference/WC}

Comparison of the highest category of WC with the lowest category revealed significant associations between greater $\mathrm{WC}$ and increased risk of total colorectal cancer. Subgroup analyses further identified the robustness of the association between abdominal obesity and $\mathrm{CRC}$ risk.

Stratifying by sex from male and female CRC, for the highest vs. lowest categories of WC level, were 1.38 (95\% CI, 1.19-1.59) and 1.44 (95\% CI, 1.28-1.61) respectively. By geographic region, the pooled RRs of CRC for the highest vs. lowest categories of WC level were 1.31 for Asia, 1.50 in the United States, and 1.29 for Europe. Meta-regression analysis showed that geographic region was the source of heterogeneity. ${ }^{1,3}$

\section{Waist-to-hip ratio/WHR}

Comparison of the highest category of WHR with the lowest category revealed significant associations between higher WHR and increased risk of total colorectal cancer. Subgroup analyses further identified the robustness of the association between abdominal obesity and $\mathrm{CRC}$ risk.

Stratifying by sex, the pooled RRs of CRC from male and female CRC studies for the highest vs. lowest categories of WHR level were 1.47 and 1.30 respectively. By geographic region, the pooled RRs of CRC for the highest vs. lowest categories of WHR level were 1.26 for Asia, 1.32 for the United States, and 1.51 for Europe. Meta-regression analysis showed that geographic region and sex were possible sources of heterogeneity. ${ }^{1,2}$

\section{Discussion}

Systematic review and meta-analysis aiming to examine the association between abdominal obesity and colorectal cancer confirmed the evidence of an increased risk of total colorectal cancer, colon cancer and rectal cancer, with greater WC and WHR.

Several mechanisms have been proposed to explain how general and central obesity enhances colorectal neoplasm risk. Previous
Volume II Issue 5 - 2020

\author{
Hajj Adel Anis \\ Cedars - Jebel Ali International Hospital, UAE
}

Correspondence: Hajj Adel Anis, Medical Oncologist, Cedars Jebel Ali International Hospital, 9370 Rue Lajeunesse, Montreal, UAE,Tel 438992 5516,Email ahajj@dr.com

Received: October 16, 2020 | Published: October 30, 2020

studies have demonstrated that the fat itself can influence CRC risk. Adipocytes and preadipocytes could promote proliferation of CRC cells. Fatty acid synthase overexpression has been shown to be associated with CRC phenotype. Obesity can change the function of essential hormones like insulin and leptin. Insulin is the hormone that regulates blood sugar and obese individuals have higher levels of insulin than those with a normal weight. This causes cell functions to change as the hormones cause irregular cell growth in the colon. Leptin is a hormone responsible for inducing a feeling of satiety and causes someone to stop eating. Levels of this hormone are up to five times higher than nonobese patients. Leptin is also responsible for cell growth in areas of the colon and, with five times as much exposure, these cells can grow uncontrollably which may create tumors., ${ }^{2,4-7}$ Moreover, this positive association exists in both men and women, different geographic regions and different anatomical sites.

\section{Conclusion}

Overweight and obesity constitute the fifth leading risk for overall mortality, accounting for at least 2.8 million adult deaths each year. Epidemiological data suggest that obesity is associated with a $30-70 \%$ increased risk of colon cancer in men, whereas the association is less consistent in women. Similar trends exist for colorectal adenoma, although the risk appears lower. ${ }^{2}$ Future research will need to focus on prospective studies with well-considered methodology in order to improve the interpretation of causality. ${ }^{1,2}$

\section{Acknowledgments}

None.

\section{Conflicts of interest}

The authors declare there are no conflicts of interest.

\section{Funding}

None.

\section{References}

1. Dai Z, Xu YC, Niu L. Obesity and colorectal cancer risk: a meta-analysis of cohort studies. World J Gastroenterol. 2007;13(31):4199-4206. 
2. Ana Silva, Gil Faria, António Araújo, et al. Impact of adiposity on staging and prognosis of colorectal cancer. Crit Rev Oncol Hematol. 2020; $145: 102857$.

3. Amalia Karahalios, Julie A Simpson, Laura Baglietto, et al. Change in weight and waist circumference and risk of colorectal cancer: results from Melbourne Collaborative Cohort Study. BMC Cancer. 2016;16:157.

4. Catherine R Guffey, Daping Fan, Udai P Singh, et al. Linking obesity to colorectal cancer: recent insights into plausible biological mechanisms. Curr Opin Clin Nutr Metab Care. 2013;16(5):595-600.
5. Silvia Riondino, Mario Roselli, Raffaele Palmirotta, et al. Obesity and colorectal cancer: role of adipokines in tumor initiation and progression. World J Gastroenterol. 2014;20(18):5177-5190.

6. Stachowicz M, Mazurek U, Nowakowska-Zajdel E, et al. Leptin and its receptors in obese patients with colorectal cancer. J Biol Regul Homeost Agents. 2010;24(3):287-295.

7. Schoen RE, Tangen CM, Kuller LH, et al. Increased blood glucose and insulin, body size, and incident colorectal cancer. J Natl Cancer Inst. 1999;91(13):1147-1154 